\title{
Autism in a case of congenital myasthenic syndrome
}

\author{
Atoosa Azarang ${ }^{1}$, Samantha C. Cohen ${ }^{1,2}$, Randi J. Hagerman ${ }^{1,2}$ and Ricardo A. Maselli ${ }^{3 *}$ \\ ${ }^{1}$ M.I.N.D. Institute, University of California-Davis Medical Center, Sacramento, California, USA \\ ${ }^{2}$ Department of Pediatrics, University of California-Davis Medical Center, Sacramento, California, USA \\ ${ }^{3}$ Department of Neurology, University of California, Davis, School of Medicine, Sacramento, California, USA
}

\begin{abstract}
Congenital Myasthenic Syndromes (CMS) are a heterogeneous group of disorders affecting neuromuscular transmission which result from mutations in genes encoding proteins located at the presynaptic, synaptic, or postsynaptic regions of the neuromuscular junction. The most frequent clinical presentation of $\mathrm{CMS}$ is generalized muscle weakness and fatigability which often involves bulbar and limb musculature.

Autism Spectrum Disorder (ASD) comprises a complex group of neuro developmental disorders that is characterized by deficit in social communication and interactions, as well as restrictive and repetitive patterns of behavior.

In this report, we present a patient with CMS and ASD. The association of CMS and ASD has previously been reported in CMS most commonly due to mutations in the Choline Acetyltransferase (CHAT) gene, and rarely due to mutations in the genes encoding the UDP-N-acetyl glucosamine-dolichyl-phosphate $\mathrm{N}$-acetylglucosamine phosphotransferase 1 (DPAGT1) enzyme and the synaptosomal-associated protein 25B (SNAP25B). These genes are widely expressed in both peripheral and central nervous system, which may explain this uncommon association. However, the gene causing CMS in our patient is expressed exclusively in muscle and has never been reported in association with ASD. Thus, alternative factors may be responsible for the rare association observed in our patient.
\end{abstract}

\section{Case report}

This patient is a 13-year old boy who is the product of intrauterine insemination from a non- consanguineous marriage. He had a normal prenatal period including normal fetal movements and a reported normal amniocentesis carried out due to advanced maternal age (39year old at the time of pregnancy). He was born at term by vaginal delivery and with normal Apgar score. However, during the neonatal period, he was found to be severely hypotonic with a poor suck, weak cry and ptosis. He has suffered from gastro-esophageal reflux disorder (GERD) and failure to thrive (FTT) since early infancy. In order to increase his caloric intake, a gastrostomy tube (GT) was placed for feeding at 4 years of age. He was diagnosed with CMS at age eight months, and pyridostigmine was started. His myasthenia has been relatively stable since 3,4-diaminopyridine (3,4-DAP) was added to pyridostigmine at age 7 .

Brain MRI and EEG at 12 years of age were both normal. He had only one episode of respiratory failure at age eight that required assisted ventilation. Since then, he has been on bi-level positive airway pressure (BiPAP) overnight during sleep.

His developmental milestones were delayed - he sat at 8 months, walked at age two and a half, and his first words were at three years of age. He demonstrated impairments in cognitive and adaptive abilities based on his scores on a range of developmental assessment tests. On the Mullen Scales of Early Learning (MSEL) published by Akshoomoff et al. [1], our patient scored less than the first percentile in all subtests at nine years of age, including gross motor performance at a 20 month level, visual reception at a 24 month level, fine motor at a 22 month level, receptive language at a 19 month level and expressive language at an 18 month level. In terms of adaptive functioning, the Vineland-II Survey Interview published by Sparrow et al. [2] conducted with his mother at age 13 years, demonstrated delay in all domains including communication skills 47, Daily Living Skills 51, Socialization 48, Motor Skills 51 and Adaptive Behavior Composite Score 48.

Results of the assessments, including observations, history and standardized measures, indicated that he met diagnostic criteria for ASD using the Diagnostic and Statistical Manual of Mental Disorders Fifth Edition (DSM 5) criteria. In addition to ASD, he demonstrated Obsessive-Compulsive Disorder (OCD), anxiety and Attention Deficit Hyperactive Disorder (ADHD), inattentive subtype. Examination at age 12 years showed that his weight was $34.156 \mathrm{~kg}$ ( $>50^{\text {th }}$ percentile), height was $1.346 \mathrm{~m}$ ( $>50^{\text {th }}$ percentile), head circumference was $0.545 \mathrm{~m}$ ( $>50^{\text {th }}$ percentile), and Body Mass Index (BMI) was 18.9 $\left(>50^{\text {th }}\right.$ percentile). He had an oval shaped face, and his ears were not prominent. He had bilateral ptosis, a high-arched palate and crowding of his teeth. He had a regular heart rate and rhythm and there were no murmurs. Abdomen was soft without hepatosplenomegaly. $\mathrm{He}$ was able to ambulate independently but often lacked stamina and was largely transported in a specially fitted stroller. He was diffusely weak, and fatigued easily. ASD symptoms were reflected in his poor ability to make eye to eye contact and lack of response to social stimulation. $\mathrm{He}$ was mainly engaged in his own thought processes and frequently made utterances that were not meaningful.

Correspondence to: Ricardo A. Maselli M.D., Neurology Department, University of California Davis, 1515 Newton court, Davis, California, USA, Tel: 1 (530) 754-5011; Fax: 1 (530) 754-5036; E-mail: ramaselli@ucdavis.edu

Key words: autism spectrum disorder, congenital myasthenic syndrome, epigenetic variation

Received: July 29, 2016; Accepted: August 12, 2016; Published: August 16, 2016 
Genetic diagnostic testing for CMS revealed that the patient was compound heterozygous for mutations in the gene encoding the $\varepsilon$ subunit of the Acetyl Choline Receptor (AChR) ion channel (CHRNE). The paternal side carries the p.Pro121 Leu mutation that has been reported in association with the low agonist binding affinity syndrome or Fast Channel Congenital Myasthenic Syndrome (FCCMS), and the other allele from the maternal side has the sequence variation c.137 $\mathrm{T}>\mathrm{C}$ that results in p.Tyr 15 His. This variation has been reported in the SNP data base (rs144169073), but has a very low minor allele frequency (0.0002) and, therefore, is likely pathogenic due to loss of expression of the $\varepsilon$ subunit.

Additional genetic studies included Chromosomal Microarray Analysis (CMA) that identified two copy number gain in chromosome bands 4q21.21 and 6q27. No deletions in the mitochondrial genome were detected. DNA analysis of the fragile $\mathrm{X}$ mental retardation1 gene (FMR1) and antibody testing for AChR were both negative. Our patient's parents and his 9 year old brother are all healthy. His brother was conceived by in vitro fertilization with pre-implantation selection of a fertilized egg that carried neither one of the mutations associated with CMS.

In addition to speech and language therapy started at age 3, our patient has been maintained on Dexmethylphenidate hydrochloride Extended Release XR (d-MPH XR, Focalin) for his ADHD symptoms since age 11. After the medicine was started he has made some improvements in the areas of language and cognition, and his level of distractibility and fidgetiness has mildly decreased. He was on a trial of low dose of sertraline for three months at age 9 without significant overall improvements, so the medication was discontinued.

\section{Discussion}

Autism Spectrum Disorder is not frequently associated with CMS; however, intellectual disabilities resulting from hypoxic encephalopathy due to sudden episodes of dyspnea/apnea and bulbar weakness are occasionally found in some cases of CMS [3-8]. Our patient did not have multiple episodes of apnea that could explain his intellectual disability.

In addition, varied degrees of intellectual impairment have been reported in patients with CMS caused by mutations in the CHAT gene, but not in CMS caused by mutations in CHRNE. Since CHAT is widely expressed in the brain and is necessary for acetylcholine re-synthesis in cholinergic neurons of the Central Nervous System (CNS) $[9,10]$, some degree of intellectual impairment is often found in patients with CMS due to CHAT mutations. By contrast, the $\varepsilon$ subunit is expressed exclusively in the peripheral nervous system; therefore autism in patients with mutations in this gene is not expected [11].

Central Nervous System involvement has also been reported in UDP-N-acetyl glucosamine-dolichyl-phosphate $\mathrm{N}$-acetylglucosaminephosphotransferase 1 (DPAGT1)-associated myasthenic syndrome, as DPAGT1 is highly expressed in muscles and brain. It is speculated that hypo glycosylation of synaptic proteins could cause defects in the CNS and at the neuromuscular junction (NMJ) [12].

More recently, a study reported that the (p.Ile 67Asn) mutation in the synaptosomal-associated protein 25B (SNAP25B) gene causes a novel presynaptic CMS associated with cortical hyper excitability, cerebellar ataxia, intellectual disability, and autism. SNAP25B is a well characterized component of the soluble N-ethylmaleimidesensitive factor attachment protein receptor (SNARE) complex, which is involved in the exocytosis of neurotransmitters at the CNS and NMJ. Thus, defects of this gene can explain the dual clinical manifestations of CMS and autism [13]. The recent explosion of genetic associations in ASD research has highlighted the diversity of the disorder's etiology [14,15]. Genomic analysis estimates that 400 to 1000 genes are involved in ASD susceptibility including CHD8, GRIN2B, and SCN2A $[16,17]$. The estimated heritability of ASD is 0.7 to 0.8 , which while relatively high leaves room for non-inherited factors, including de novo mutations, epigenetic variations, and environmental factors leading to a complex risk architecture [18]. At this point, it is clear that rare microscopically detectable chromosomal rearrangements and submicroscopic deletions or duplications such as Copy Number Variants (CNVs) contribute to risk of ASD. These variants can be inherited from unaffected parents or arise as de novo mutations during the meiotic divisions of gametogenesis. Significant enrichment in the rate of de novo CNVs is observed in individuals with ASDs. Furthermore, CNVs in specific regions of the genome including $15 q 11-13,16 \mathrm{p} 11.2$, and 22q11.2 are associated with increased risk for ASDs [19-22]. In our case, the two copy number gain in chromosome bands $4 \mathrm{q} 21.21$ and $6 \mathrm{q} 27$ were not felt to contribute to the risk of ASD. Our patient is the product of medically assisted conception and these interventions, including intrauterine insemination, can be associated with low but significant increased risk for ASD and intellectual disability [23-26]. According to the developmental origin of the health and disease hypothesis, environmental factors including those used in medically assisted conception methods, such as culture media and handling procedures, which act during early embryonic development, could interact with the genotype and change the capacity of the organism to cope with other environment factors later in life [27]. The early development of the human nervous system is a complex and neatly orchestrated process that can be easily affected by these invasive interventions [28]. Nevertheless, possible neurodevelopmental deficits in children born after medical assisted procedures may be related to several non-procedural factors including the underlying sub-fertility or other conditions in the parents e.g. men with low sperm quality are more likely to have chromosomal abnormalities that they may pass on to their offspring. There may be multiple additive effects leading to ASD in our patient including his CNV plus the intrauterine insemination, as well as some potential additional effects of the CMS mutation previously not reported [29,30].

Our patient made some improvement in speech and cognition after stimulant treatment which improved his school performance, but did not cure his ASD or ID.

In summary, we present a case of CMS with associated features of ASD who is the product of intrauterine insemination. While the genetic basis of CMS in our patient is certain, the genetic causes of his ASD are unclear and may be multifactorial. It is possible that the association of CMS and ASD in our patient was fortuitous, yet still raises questions about a complex interplay of genetic and epigenetic factors leading to the manifestation of our patient's rare phenotype.

\section{Funding}

This report was supported by a grant from the UCEED Health and Human Services Administration of Developmental Disabilities (Grant 90DD0596) and the MIND Institute Intellectual and Developmental Disability Center (IDDRC; U54HD079125). We also thank the family who has supported our case report. 


\section{Conflict of interest}

The authors have no conflict of interest to declare.

\section{References}

1. Akshoomoff N (2006) Use of the Mullen Scales of Early Learning for the assessment of young children with Autism Spectrum Disorders. Child Neuropsychol 12: 269-277. [Crossref]

2. Sparrow SS, Cicchetti D, Balla D (2005) Vineland adaptive behavior scales. (2ndedn) (Vineland-II). Circle Pines, MN: AGC Publishing.

3. Rinz CJ, lennon VA, James F, Thoreson JB, Tsai k, et al. (2015) A CHRNE frameshift mutation causes congenital myasthenic syndrome in young Jack Russell Terriers. Neuromuscul Disord 25: 921-927. [Crossref]

4. Kinali M, Beeson D, Pitt MC, Jungbluth H, Simonds AK, et al. (2008) Congenitalmyasthenic syndromes in childhood: diagnostic and management challenges. J Neuroimmunol 201-202: 6-12.[Crossref]

5. Engel AG, Shen XM, Selcen D, Sine SM (2015) Congenitalmyasthenic syndromes: pathogenesis, diagnosis, and treatment. Lancet Neurol 14: 461.[Crossref]

6. Engel AG1 (2008) Congenital myasthenic syndromes. Handb Clin Neurol 91: 285-331. [Crossref]

7. Webster R, Liu WW, Chaouch A, Lochmüller H, Beeson D (2014) Fast-channel congenital myasthenic syndrome with a novel acetylcholine receptor mutation at the a-e subunit interface. Neuromuscul Disord 24: 143-147. [Crossref]

8. Azuma Y, Nakata T, Tanaka M, Shen XM, Ito M, et al. (2015) Congenital myasthenic syndrome in Japan: ethnically unique mutations in muscle nicotinic acetylcholine receptor subunits. Neuromuscul Disord 25: 60-69.[Crossref]

9. Engel AG, Shen XM, Selcen D, Sine S (2012) New horizons for congenital myasthenic syndromes. Ann N Y Acad Sci 1275: 54-62.[Crossref]

10. Schara U, Christen HJ, Durmus H, Hietala M, Krabetz K, et al. (2010) Long-term follow-up in patients with congenital myasthenic syndrome due to CHAT mutations. Eur J Paediatr Neurol 14: 326-333. [Crossref]

11. Levin ED1 (2002) Nicotinic receptor subtypes and cognitive function. $J$ Neurobiol 53 : 633-640.[Crossref]

12. Selcen D, Shen XM, Brengman J, Li Y, Stans AA, et al. (2014) DPAGT1 myasthenia and myopathy: genetic, phenotypic, and expression studies. Neurology 82:1822-30. [Crossref]

13. Shen XM, Selcen D, Brengman J, Engel AG (2014) Mutant SNAP25B causes myasthenia, cortical hyperexcitability, ataxia, and intellectual disability. Neurology 83 : 2247-2255.[Crossref]

14. Robinson EB, Neale BM, Hyman SE (2015) Genetic research in autism spectrum disorders. Curr Opin Pediatr 27: 685-691.[Crossref]
15. Schanen NC (2006) Epigenetics of autism spectrum disorders. Hum Mol Genet 15 Spec No 2: R138-150.[Crossref]

16. Sahin M, Sur M (2015) Genes, circuits, and precision therapies for autism and related neuro developmental disorders. Science 350.[Crossref]

17. Gonzalez-Gronow M, Cuchacovich M, Francos R, Cuchacovich S, Blanco A, et al (2015) Catalytic autoantibodies against myelin basic protein (MBP) isolated from serum of autistic children impair in vitro models of synaptic plasticity in rat hippocampus. $J$ Neuroimmunol 287:1-8. [Crossref]

18. Lord C, Bishop S, Anderson D (2015) Developmental trajectories as autism phenotypes. Am J Med Genet C Semin Med Genet 169: 198-208.[Crossref]

19. Sanders SJ, Ercan-Sencicek AG, Hus V, Luo R, Murtha MT, et al. (2011) Multiple recurrent de novo CNVs, including duplications of the $7 \mathrm{q} 11.23$ Williams syndrome region, are strongly associated with autism. Neuron 70: 863-885. [Crossref]

20. De Rubeis S1, Buxbaum JD2 (2015) Genetics and genomics of autism spectrum disorder: embracing complexity. Hum Mol Genet 24: R24-31.[Crossref]

21. Levy D, Ronemus M, Yamrom B, Lee YH, Leotta A, et al. (2011) Rare de novo and transmitted copy-number variation in autistic spectrum disorders. Neuron 70: 886-897. [Crossref]

22. Krumm N, Turner TN, Baker C, Vives L, Mohajeri K, et al. (2015) Excess of rare, inherited truncating mutations in autism. Nat Genet 47: 582-588.[Crossref]

23. Zhan QT, Pan PP, Xu XR, Lou HY, Lou YY, et al. (2013) An overview of studies on psychological well-being in children born following assisted reproductive technologies. J Zhejiang Univ Sci B 14: 947-960.[Crossref]

24. Bay B, Mortensen EL, Hvidtjørn D, Kesmodel US (2013). Fertility treatment and risk of childhood and adolescent mental disorders: register based cohort study. BMJ 347: f3978.

25. Sandin S1, Nygren KG, Iliadou A, Hultman CM, Reichenberg A (2013) Autism and mental retardation among offspring born after in vitro fertilization. JAMA 310: 75-84 [Crossref]

26. Gluckman PD, Hanson MA, Cooper C, Thornburg KL (2008) Effect of in utero and early-life conditions on adult health and disease. NEngl J Med 359: 61-73.[Crossref]

27. deGraaf-Peters VB1, Hadders-Algra M (2006) Ontogeny of the human central nervous system: what is happening when? Early Hum Dev 82: 257-266.[Crossref]

28. Felch AC, Granger RH (2008)Thehypergeometric connectivity hypothesis: divergen performance of brain circuits with different synaptic connectivity distributions. Brain Res 1202: 3-13. [Crossref]

29. Sazonova A, Källen K, Thurin-Kjellberg A, Wennerholm UB, Bergh C (2011) Factors affecting obstetric outcome of singletons born after IVF. Hum Reprod 26: 2878-2886. [Crossref]

30. Magli MC, Gianaroli L, Ferraretti AP, Gordts S, Fredericks V, et al. (2009) Paternal contribution to aneuploidy in preimplantation embryos. Reprod Biomed Online 18 536-542.[Crossref]

Copyright: (C2016 Azarang A. This is an open-access article distributed under the terms of the Creative Commons Attribution License, which permits unrestricted use, distribution, and reproduction in any medium, provided the original author and source are credited. 